\title{
Chapter 77 \\ Report on the Global Environment \\ Competitiveness of Kenya
}

Kenya is located in Eastern Africa, borders on Somalia, Tanzania, Ethiopia and other countries, and the southeast closes to the Indian Ocean. It mainly is the Savannah climate and the tropical monsoon, which lacks of mineral resources. It covers 569 thousand square kilometres. It had a population of 41.6 million and domestic production

Score: 47.76

Rank:

$\mathbf{9 0}$ the gross (GDP) of USD 33.6 billion in 2011. Through the evaluation of global environment competitiveness, we can know that the environment competitiveness index of Kenya ranks at 90 among 133 countries.

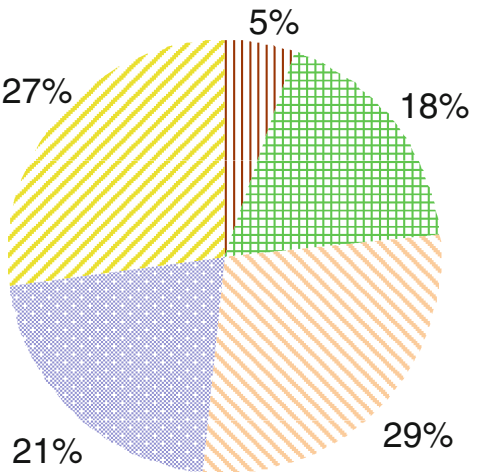

Fig. 77.1 Contribution of sub-index of GEC
III Resource

Environment

Competitiveness

\# Ecological

Environment

Competitiveness

Environment

Carrying

Competitiveness

- Environment

Management

Competitiveness

Environment

Harmony

Competitiveness 


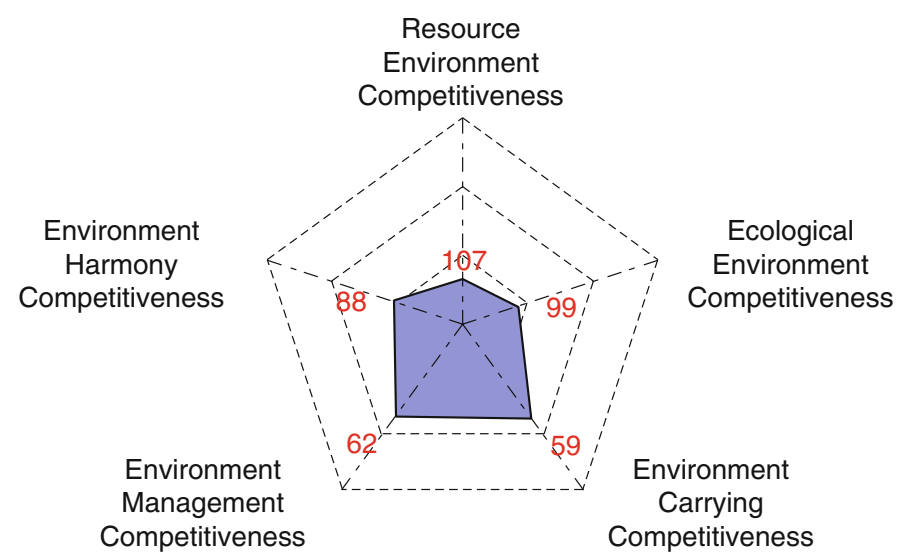

Fig. 77.2 Rank of sub-index of GEC

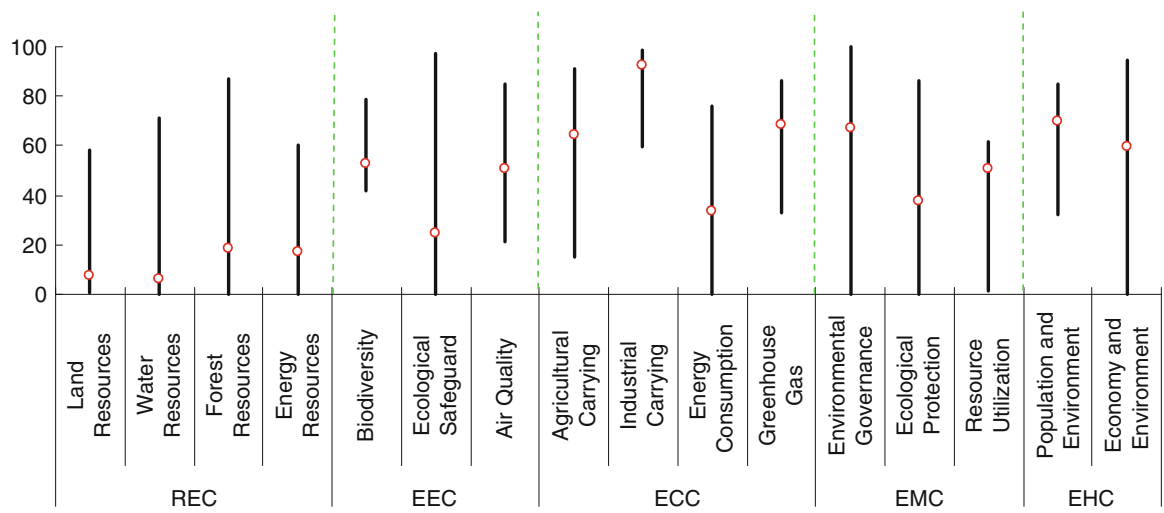

Fig. 77.3 Score and rank of the pillars of GEC

Table 77.1 Score and rank of all indicators of GEC

\begin{tabular}{|c|c|c|c|c|c|}
\hline Indicators & Score & Rank & Indicators & Score & Rank \\
\hline 1 Resource Environment & 12.31 & 107 & Groundwater & 0.84 & 110 \\
\hline Competitiveness & & & \multirow{2}{*}{$\begin{array}{l}\text { Total internal renewable } \\
\text { water resources }\end{array}$} & \multirow{2}{*}{1.83} & \multirow[t]{2}{*}{110} \\
\hline 1.1 Land Resources & 7.71 & 104 & & & \\
\hline Land area per capita & 2.44 & 66 & 1.3 Forest Resources & 18.59 & 107 \\
\hline $\begin{array}{l}\text { Percentage of arable land to } \\
\text { total land area }\end{array}$ & 16.26 & 82 & $\begin{array}{l}\text { Growing stock in forest and } \\
\text { other wooded land }\end{array}$ & 51.91 & 54 \\
\hline Arable land per capita & 6.18 & 80 & \multirow{2}{*}{$\begin{array}{l}\text { Proportion of land area } \\
\text { covered by forest }\end{array}$} & \multirow[t]{2}{*}{7.11} & \multirow[t]{2}{*}{109} \\
\hline 1.2 Water Resources & 6.26 & 105 & & & \\
\hline Surface water & 0.57 & 108 & Forest area per capita & 0.58 & 101 \\
\hline Annual precipitation & 21.78 & 87 & 1.4 Energy Resources & 17.26 & 24 \\
\hline
\end{tabular}


Table 77.1 (continued)

\begin{tabular}{|c|c|c|c|c|c|}
\hline Indicators & Score & Rank & Indicators & Score & Rank \\
\hline Fossil energy & 0.00 & 64 & \multirow{2}{*}{$\begin{array}{l}\text { Energy consumption per unit } \\
\text { of land area }\end{array}$} & \multirow[t]{2}{*}{99.93} & \multirow[t]{2}{*}{36} \\
\hline Energy production & 0.39 & 95 & & & \\
\hline $\begin{array}{l}\text { Proportion of combustible } \\
\text { renewables and waste to }\end{array}$ & 77.95 & 11 & $\begin{array}{l}\text { Ratio of clean energy } \\
\text { consumption }\end{array}$ & 12.20 & 45 \\
\hline total energy consumption & & & Elasticity of energy & 13.29 & 102 \\
\hline $\begin{array}{l}\text { Net energy imports of the } \\
\text { energy consumption }\end{array}$ & 10.20 & 57 & \multirow{2}{*}{$\begin{array}{l}\text { Elasticity of electric power } \\
\text { consumption }\end{array}$} & 8.10 & \multirow[t]{2}{*}{113} \\
\hline $\begin{array}{l}2 \text { Ecological Environment } \\
\text { Competitiveness }\end{array}$ & 43.56 & 99 & & 68.27 & \\
\hline 2.1 Biodiversity & 52.59 & 120 & \multirow{2}{*}{$\begin{array}{l}\text { Growth rate of } \mathrm{CO}_{2} \\
\text { emissions }\end{array}$} & \multirow[t]{2}{*}{45.88} & \multirow[t]{2}{*}{88} \\
\hline Threatened fish species & 67.92 & 116 & & & \\
\hline Threatened mammal species & 84.78 & 106 & \multirow{2}{*}{$\begin{array}{l}\text { Growth rate of Methane } \\
\text { emissions }\end{array}$} & \multirow[t]{2}{*}{61.00} & \multirow[t]{2}{*}{48} \\
\hline Threatened plant species & 92.65 & 112 & & & \\
\hline $\begin{array}{l}\text { GEF benefits index for } \\
\text { biodiversity }\end{array}$ & 8.80 & 31 & $\begin{array}{l}\mathrm{CO}_{2} \text { emissions per unit of } \\
\text { land area }\end{array}$ & 99.98 & 22 \\
\hline 2.2 Ecological Safeguard & 24.60 & 58 & \multirow{2}{*}{$\begin{array}{c}\mathrm{CO}_{2} \text { emissions per unit of } \\
\text { energy consumption }\end{array}$} & \multirow[t]{2}{*}{88.59} & \multirow[t]{2}{*}{8} \\
\hline Terrestrial protected areas & 31.79 & 61 & & & \\
\hline Marine protected areas & 13.81 & 24 & \multirow{2}{*}{$\begin{array}{l}4 \text { Environment Management } \\
\text { Competitiveness }\end{array}$} & \multirow[t]{2}{*}{50.56} & \multirow[t]{2}{*}{62} \\
\hline 2.3 Air Quality & 51.02 & 91 & & & \\
\hline Inhalable particles (PM10) & 78.10 & 69 & \multirow{2}{*}{$\begin{array}{l}\text { 4.1 Environmental } \\
\text { Governance }\end{array}$} & \multirow[t]{2}{*}{67.00} & \multirow[t]{2}{*}{112} \\
\hline Particulate matter (PM2.5) & 89.98 & 35 & & & \\
\hline Index of indoor air pollution & 4.20 & 108 & $\begin{array}{l}\text { Agricultural chemicals } \\
\text { regulation }\end{array}$ & N/A & N/A \\
\hline Nitrogen oxides emission & 66.76 & 88 & \multirow{3}{*}{$\begin{array}{l}\text { Percentage of the rural } \\
\text { population with access to } \\
\text { an improved water } \\
\text { source }\end{array}$} & \multirow{3}{*}{52.00} & \multirow{3}{*}{104} \\
\hline Sulfur dioxide emission & 40.84 & 45 & & & \\
\hline $\begin{array}{l}3 \text { Environment Carrying } \\
\text { Competitiveness }\end{array}$ & 67.43 & 59 & & & \\
\hline 3.1 Agricultural Carrying & 64.63 & 87 & \multirow{2}{*}{$\begin{array}{l}\text { Percentage of the urban } \\
\text { population with access to } \\
\text { an improved water }\end{array}$} & 82.00 & 122 \\
\hline $\begin{array}{l}\text { Cereal yield per unit of } \\
\text { arable land }\end{array}$ & 14.00 & 102 & & & \\
\hline Fertilizer consumption per & 97.38 & 48 & source & & \\
\hline unit of arable land & & & 4.2 Ecological Protection & 37.91 & 49 \\
\hline $\begin{array}{l}\text { Annual freshwater } \\
\text { withdrawals for }\end{array}$ & 99.40 & 49 & $\begin{array}{l}\text { Area of plantation and } \\
\text { afforestation }\end{array}$ & 0.26 & 68 \\
\hline agriculture per unit of & & & Biome protect & 67.20 & 57 \\
\hline 3.2 Industrial Carrying & 92.36 & 55 & $\begin{array}{l}\text { Overfishing of fishing } \\
\text { resources }\end{array}$ & 58.82 & 35 \\
\hline Net exports as a percentage & 81.82 & 64 & 4.3 Resource Utilization & 50.99 & 12 \\
\hline $\begin{array}{l}\text { of GDP } \\
\text { Electric power consumption }\end{array}$ & 90.53 & 76 & $\begin{array}{l}\text { Utilization rate of water } \\
\text { resources }\end{array}$ & 0.36 & 64 \\
\hline $\begin{array}{l}\text { per unit of value added } \\
\text { of industry }\end{array}$ & & & $\begin{array}{l}\text { Percentage of total internal } \\
\text { renewable water }\end{array}$ & 61.42 & 71 \\
\hline $\begin{array}{l}\mathrm{SO}_{2} \text { emissions per unit of } \\
\text { value added of industry }\end{array}$ & 99.68 & 82 & $\begin{array}{l}\text { resources to total water } \\
\text { resources }\end{array}$ & & \\
\hline $\begin{array}{l}\text { Annual freshwater } \\
\text { withdrawals for industry }\end{array}$ & 97.41 & 66 & $\begin{array}{c}\text { Percentage of agricultural } \\
\text { land to total land area }\end{array}$ & 56.80 & 55 \\
\hline $\begin{array}{l}\text { per value added of } \\
\text { industry }\end{array}$ & & & $\begin{array}{l}\text { Percentage of fossil fuel } \\
\text { energy consumption to }\end{array}$ & 85.37 & 8 \\
\hline 3.3 Energy Consumption & 33.38 & 65 & total energy consumption & & \\
\hline
\end{tabular}


Table 77.1 (continued)

\begin{tabular}{|c|c|c|c|c|c|}
\hline Indicators & Score & Rank & Indicators & Score & Rank \\
\hline $\begin{array}{l}5 \text { Environment Harmony } \\
\text { Competitiveness }\end{array}$ & 64.93 & 88 & $\begin{array}{l}\mathrm{CO}_{2} \text { emissions (metric tons } \\
\text { per capita) }\end{array}$ & 99.38 & 16 \\
\hline $\begin{array}{l}\text { 5.1 Population and } \\
\text { Environment }\end{array}$ & 70.19 & 67 & $\begin{array}{l}\text { Energy consumption per } \\
\text { capita }\end{array}$ & 97.30 & 21 \\
\hline \multirow{2}{*}{$\begin{array}{l}\text { Improved sanitation facilities } \\
\text { (\% of population with } \\
\text { access) }\end{array}$} & 31.00 & 111 & $\begin{array}{l}\text { 5.2 Economy and } \\
\text { Environment }\end{array}$ & 59.67 & 92 \\
\hline & 97.41 & 25 & $\begin{array}{l}\text { Land resource utilization } \\
\text { efficiency }\end{array}$ & 0.02 & 112 \\
\hline $\begin{array}{l}\text { Motor vehicles (per 1,000 } \\
\text { people) }\end{array}$ & 0.60 & 114 & $\begin{array}{l}\text { Sulfur dioxide emissions per } \\
\text { unit of GDP }\end{array}$ & 94.60 & 67 \\
\hline $\begin{array}{l}\text { Renewable internal } \\
\text { freshwater resources per } \\
\text { capita }\end{array}$ & & & $\begin{array}{l}\text { Carbon dioxide emissions } \\
\text { per unit of GDP }\end{array}$ & 89.07 & 62 \\
\hline $\begin{array}{l}\mathrm{SO}_{2} \text { emissions (metric tons } \\
\text { per capita) }\end{array}$ & 99.44 & 16 & $\begin{array}{l}\text { Energy consumption per unit } \\
\text { of GDP }\end{array}$ & 55.00 & 116 \\
\hline
\end{tabular}

Table 77.2 Rank distribution of the individual indicators of GEC

\begin{tabular}{lllllll}
\hline & $\begin{array}{l}\text { Number } \\
\text { of the } \\
\text { individual } \\
\text { indicators }\end{array}$ & $\begin{array}{l}\text { Rank } \\
1-10\end{array}$ & $\begin{array}{l}\text { Rank } \\
11-30\end{array}$ & $\begin{array}{l}\text { Rank } \\
31-60\end{array}$ & $\begin{array}{l}\text { Rank } \\
61-100\end{array}$ & $\begin{array}{l}\text { Rank } \\
101-133\end{array}$ \\
\hline Sub-index & 14 & 0 & 2 & 1 & 5 & 6 \\
\hline $\begin{array}{c}\text { Resource Environment } \\
\quad \text { Competitiveness }\end{array}$ & 11 & 0 & 0 & 2 & 4 & 5 \\
$\begin{array}{c}\text { Ecological Environment } \\
\quad \text { Competitiveness }\end{array}$ & 15 & 0 & 2 & 5 & 6 & 2 \\
$\begin{array}{c}\text { Environment Carrying } \\
\quad \text { Competitiveness }\end{array}$ & 10 & 0 & 1 & 3 & 3 & 2 \\
$\begin{array}{c}\text { Environment Management } \\
\quad \text { Competitiveness }\end{array}$ & 10 & 0 & 3 & 0 & 4 & 3 \\
$\begin{array}{c}\text { Environment Harmony } \\
\text { Competitiveness }\end{array}$ & 60 & 0 & 8 & 11 & 22 & 18 \\
\hline Total & & & & & & \\
\hline
\end{tabular}

Open Access This chapter is distributed under the terms of the Creative Commons Attribution Noncommercial License, which permits any noncommercial use, distribution, and reproduction in any medium, provided the original author(s) and source are credited. 